\title{
NATURE ET HUMANITÉ: ÉVOLUTION DE CES CONCEPTS DANS L'EUVRE DE MAURICE MERLEAU-PONTY D’APRÈS ÉTIENNE BIMBENET
}

\author{
NATURALEZA Y HUMANIDAD: EVOLUCIÓN DE ESTOS CONCEPTOS EN LA \\ OBRA DE MAURICE MERLEAU-PONTY SEGÚN ÉTIENNE BIMBENET
}

\author{
Stéphanie Perruchoud ${ }^{1}$ \\ Universitat Internacional de Catalunya (España)
}

Recibido: 03-05-2014

Aceptado: 10-09-2014

Résumé: Le thème de prédilection dans la phase post Deuxième Guerre Mondiale, en France, a certainement été celui de l'homme et de sa place dans la société. Toutefois, Merleau-Ponty proposera, et ceci dès le commencement, une réflexion anthropologique profonde. Que signifie être humain pour l'homme déjà au niveau biologique? Dans la Structure du comportement, le philosophe français invite à commencer toute réflexion par le bas. De cette manière, les gestes les plus basiques, les réflexes, sont indicateurs d'un être-au-monde. Dans le cas de l'homme, cet être-au-monde se réalise au fur et à mesure de l'apparition de ses différentes structures. Ainsi, l'attitude catégoriale est orientée par le domaine physique et vital mais les sublime en leur donnant un nouveau sens. Etienne Bimbenet, dans un livre intitulé Nature et humanité, soutient que la façon dont Merleau-Ponty a posé le problème homme-nature devait nécessairement se résoudre par un abandon du thème anthropologique et une ouverture à une philosophie de la nature. Dans cette présentation, nous tacherons de présenter l'argumentation de Bimbenet à partir d'une perspective critique.

Mots-clés: Nature, humanité, anthropologie, comportement, corps-esprit

[1] (stephanie.perruchoud@gmail.com) Doctoranda y becaria de la Universidad Internacional de Catalunya. Actualmente está realizando una estancia de investigación en Instituto de ética y Derechos humano de la Universidad de Friburgo sobre el tema del cuerpo vulnerable. Perteneciente al grupo de investigación: "Anthropology of corporeality / Interdisciplinary studies in embodied subjectivity”; Investigador principal: Dr. Xavier Escribano; y entidad financiadora: Universitat Internacional de Catalunya. 
Resumen: El tema de predilección durante la fase post Segunda Guerra Mundial en Francia ha sido sin duda el del hombre y de su lugar en la sociedad. Sin embargo, Merleau-Ponty propondrá, desde el inicio de su obra, una reflexión antropológica profunda. ¿Qué significa ser humano para el hombre a un nivel meramente biológico? En la Estructura del comportamiento, el filósofo francés invita a empezar toda reflexión desde abajo. De esta manera, los gestos más básicos, los reflejos, son los indicadores de un estar-en-el mundo. En el caso del hombre, este estar-en-el mundo se realiza con la aparición de sus distintas estructuras. La actitud categorial, pues, está ciertamente orientada por el ámbito físico y vital pero los sublima dándoles un nuevo sentido. Etienne Bimbenet, en su libro Nature et humanité, sostiene que la forma en la que Merleau-Ponty expuso el problema hombre-naturaleza tenía que desembocar necesariamente en un abandono del tema antropológico y en una apertura a una filosofía de la naturaleza. En este trabajo nos dedicaremos a presentar el argumento de Bimbenet desde una perspectiva crítica.

Palabras-clave: Naturaleza, humanidad, antropología, comportamiento, cuerpo-espíritu

Étienne Bimbenet: Nature et humanité. Le problème anthropologique dans l'œuvre de Merleau-Ponty. Paris : Vrin, 2004. 322 páginas.

Tout est fabriqué et tout est naturel chez l'homme

Maurice Merleau-Ponty

Cette phrase tirée de la Phénoménologie de la perception résume parfaitement la ligne de recherche de Maurice Merleau-Ponty, mais aussi la tâche qu'il a assumée comme penseur : celle de vouer ses efforts à parler du problème de l'homme, de sa provenance, de son histoire, de son existence. Une véritable anthropologie philosophique devrait toujours débuter par une reconnaissance de la part de mystère qui recouvre l'être humain et, à posteriori, par un refus d'asserter avec précipitation. La méthode phénoménologique utilisée dans l'ensemble de l'œuvre Pontienne comme méthode philosophique pour traiter ce grand thème de l'humanité propose une suspension du jugement en vue d'une connaissance plus réaliste et concrète. Toute certitude doit alors laisser place à l'étonnement. Qu'est-ce que l'homme ? Quelle relation garde-t-il avec la nature qui l'entoure ? Il n'a jamais été question de résoudre un problème, sinon de le décrire et ceci de la meilleure manière possible. En ce sens, d'accord avec les différentes dimensions de son être, l'homme est perçu comme 
Nature et humanité : évolution de ces concepts dans l'œuvre de Maurice Merleau-Ponty d'après Étienne Bimbenet

totalement fabriqué [-culturel] et totalement naturel. En d'autres mots, il se crée lui-même et son milieu, notamment grâce à ses capacités intellectuelles, mais sa vie reste toujours une vie incarnée, ancrée au monde naturel par la corporéité. Il va s'en dire que Merleau-Ponty a cette façon bien à lui de comprendre certains concepts comme celui d'humanité ou de nature et c'est cela même qui interpelle tant de philosophes depuis 53 ans $^{2}$. Déjà Jean-Paul Sartre le signifiait dans un hommage écrit en son honneur dans la revue Temps Moderne [Merleau-Ponty : vivant]. Mentionnant une de leur dernière rencontre, il se souvient que Merleau-Ponty avait l'intention de publier un ouvrage sur la nature qu'il considérait, à l'instar de Whitehead, un être en haillons. Travaillant, à l'époque sur le matérialisme dialectique, la nature, pour Sartre, n'était que le résultat d'une série de relations physico-chimiques. "Je le quittai sans avoir compris ". Ce n'est que bien plus tard qu'il prit conscience que pour son ancien camarade le mot "Nature " faisait référence à une toute autre réalité : au monde sensible, universel où nous rencontrons les choses et les bêtes, notre propre corps et les autres.

Dans l'actualité, d'autres personnes tels qu'Étienne Bimbenet se sont penchés sur les concepts de Nature et Humanité pour en connaître l'ampleur et l'importance dans la pensée du phénoménologue français. Étienne Bimbenet, ancien élève de l'Ecole Normale Supérieure et Maître de Conférences à l'Université Jean Moulin-Lyon III nous offre ainsi, au travers de l'ouvrage que nous allons commenter [Nature et humanité-Le problème anthropologique dans l'œuvre de Merleau-Ponty], fruit de sa thèse et de ses recherches personnelles, non seulement un regard expert et approfondi des thèmes susmentionnés, mais également une vue panoramique de l'ensemble des textes de Merleau-Ponty. La thèse de ce texte est la constatation de l'effacement progressif et nécessaire ${ }^{3}$ $\mathrm{du}$ thème anthropologique au profit du thème de la Nature. Nous manifestons toutefois une retenue quant à l'expression de cette nécessité qui, nous semblet-il, n'est pas évidente à priori. Certes, dès la Structure du Comportement, même dès la première ligne du texte, le concept de nature apparaît comme un élément-clé et révélateur d'un intérêt orienté : Notre but est de comprendre les rapports de la conscience et de la nature ${ }^{4}$. Or il est aisé de reconnaître que ce terme a évolué chez Ponty non seulement dans son contenu sémantique, mais aussi et surtout dans l'ampleur qu'il a fini par occuper. Nous préférons ainsi dire que la Nature, découverte de manière nouvelle au travers des écrits d'un

[2] Nous rappelons que Maurice Merleau-Ponty est décédé prématurément à l'âge de 53 ans. Professeur au Collège de France et auteur de nombreux ouvrages tels que Phénoménologie de la Perception ou Signes, le légat intellectuel de ce dernier est indéniable et tous s'accordent pour dire que ce philosophe était alors à l'apogée de sa carrière.

[3] Selon Bimbenet, l'orientation des recherches de Merleau-Ponty ne pouvait qu'aboutir à l'éloignement de l'intérêt anthropologique au profit de la Nature.

[4] Maurice, Merleau-Ponty, "La structure du comportement", éd. Quadrige/Puf, Paris, 1942, p.1.

THÉMATA. Revista de Filosofía, No51 enero-junio (2015) pp.: 443-454

doi: 10.12795/themata.2015.i51.25 
Whitehead ou d'un Buytendijk, s'est présentée [plutôt qu'imposée] comme une nouvelle problématique digne d'étude ${ }^{5}$.

Dans Nature et humanité, l'anthropologie est donc présentée comme point d'ancrage d'une philosophie qu'on pourrait définir plus justement comme philosophie de la vie ou de l'existence. Et bien que l'œuvre pontienne dans son ensemble paraisse marquer une cohérence logique et une ascension progressive, Bimbenet distingue, à juste titre, deux période décisives : une première, qui occupe les deux tiers de son livre et comprend les deux premiers travaux de Merleau-Ponty [à savoir La structure du comportement et la Phénoménologie de la perception] où l'on adopte la méthode du spectateur étranger, d'une part, et la méthode réflexive, de l'autre - et une deuxième, de caractère plus ontologique, qui marque un tournant à partir de la Prose du monde. Nous tacherons de mettre en exergue les éléments les plus significatifs de ces deux temps. Pour ce faire, nous allons commencer par attribuer un nom à chaque partie : la première, que nous appellerons "Anthropologie structurelle " et la deuxième, " expression et mort de l'homme».

\section{Anthropologie structurelle}

Avant d'entrer dans l'analyse de l'œuvre, nous allons contextualiser brièvement, avec Bimbenet, l'époque dans laquelle s'inscrit le projet de MerleauPonty. La tradition philosophique et les diverses sciences portant sur l'homme ont distingué en ce dernier deux dimensions : une dimension biologique qui, sans aucun doute, le classifierait définitivement comme membre à part entière de la nature et une dimension intellectuelle, qui l'en différencierait spécifiquement. Cette vision ambigüe de l'être humain deviendra l'une des causes du divorce entre philosophie et science au XIXe siècle créant ainsi un double mythe : le mythe de la science (positivisme, mécanicisme) et le mythe philosophique (intellectualisme, matérialisme). Témoin de ce grand écart, Merleau-Ponty dénonce, de manière toujours argumentée, tout point de vue extrême et, finalement, injustifié. Le point de départ de la recherche est donc la réduction phénoménologique. Il s'agit de revenir à l'expérience muette, au mystère de l'union vécue. Ce retour originel est marqué par un refus de partager l'homme en deux secteurs (la nature d'une part et la conscience de l'autre). La philosophie de ce phénoménologue est certes critique, mais avant tout concrète et unificatrice. Tout est humain en l'homme que ce soit au niveau biologique ou de la raison. L'effort du philosophe est donc celui de tendre vers une réconciliation nature-esprit.

[5] Merleau-Ponty s'inscrit dans un courant et une période historique pour lesquels l'humanisme résonne d'une manière nouvelle et particulière. Peut-être que la traversée des deux Grandes Guerres et la terreur qui en a découlée a favorisé une approche plus pudique, plus modeste, plus incarnée de l'être humain. Il n'en reste pas moins que Ponty a toujours démontré un intérêt pour "l'homme» notamment au travers des cours donnés sur la psychologie.

THÉMATA. Revista de Filosofía, No51 enero-junio (2015) pp.: 443-454 doi: 10.12795/themata.2015.i51.25 
Nature et humanité : évolution de ces concepts dans l'œuvre de Maurice Merleau-Ponty d'après Étienne Bimbenet

Telle est la pierre d'achoppement à laquelle souhaite faire face MerleauPonty et c'est pourquoi, La structure du comportement, œuvre préparatoire à la Phénoménologie de la perception, incarne cet idéal. Comme bon investigateur, Merleau-Ponty prend le temps de discuter avec les diverses sciences de son époque, surtout avec la physiologie et la psychologie moderne. Il reconnaît en elles, le mérite de vouloir parler du vivant et de l'homme. Toutefois, il met en garde, et d'une certaine manière dénonce, contre le légat mécaniciste qui offre une méthode qui se dit certes unificatrice, mais qui devient par là aussi simplificatrice et réductionniste. La distinction qui a pu se faire dans l'Antiquité entre le corps et l'âme, entre matière et forme, trouve son apogée dans la philosophie de Descartes, pour qui, non seulement il y a lieu de séparer ces deux niveaux, mais également de choisir une méthode de recherche plus adéquate que celles de ces prédécesseurs et qui s'appuierait fondamentalement sur les causes matérielles et efficientes ${ }^{6}$. L'âme, chez Descartes, est plus connue que le corps. Grâce au doute méthodique, il est parvenu à l'élément le plus clair et distinct de l'homme : son esprit. Je peux douter de tout, mais jamais du fait que je doute, c'est-à-dire que je pense. Et pour garantir la véracité de tout ce dont nous avions mis en doute, il ne peut recourir à d'autres principes qu'à celui de Dieu? ${ }^{7}$. Ce principe va se convertir en pilier fondamental de toute science et de toute métaphysique. Il convient alors de commencer notre étude par la manière dont on connaît les choses prenant en compte l'origine de cette faculté :

«Premièrement, j’ai tâché de trouver en général les principes ou premières causes de tout ce qui est [...], sans rien considéré pour cet effet que Dieu seul qui l'a créé, ni les tirer d'ailleurs que de certaines semences de vérités qui sont naturellement en nos âmes. [...] Il me semble par là que j'ai trouvé des cieux... ${ }^{\text {" }}$

Cette recherche a été contestée par des auteurs comme Maritain qui n'ont pas hésité à qualifier la philosophie de Descartes d'angélisme, mais aussi par d'autres auteurs comme Charles Péguy qui l'ont jugé chevaleresque:

«Et bien je dis : qu'importe. Nous savons bien qu'il ne les a pas trouvés, les cieux. [...] Il veut avoir déduit tout cela, et Dieu même, à peine en passant par les principes ou premières causes, à peine en s'aidant des idées innées, de ces certaines semences de vérités

[6] Cette nouvelle méthode se caractérise d'abord par un rejet de la cause finale d'Aristote. Dieu a établi certaines lois dans la Nature, tel l'horloger qui fabrique une montre et il a donné à l'homme la capacité de les connaître, mais pas de comprendre le pourquoi final. Dans cette perspective, seule une science qui repose sur des causes efficientes et matérielles est légitime.

[7] Notons que pour démontrer l'existence de Dieu, Descartes fait appel aux pensées humaines. Ce qui revient, d'une part, à relativiser l'existence de Dieu à l'existence humaine et, d'autre part, à assurer l'existence de l'homme [ainsi que toute création] par l'existence de Dieu. Selon Kant, dans la Critique de la faculté de juger, il s'agit d'une pétition de principe ou d'une antinomie inacceptable lorsqu'on veut fonder un système de pensée cohérent.

[8] Ref : Geneviève Rodis-Lewis, "Descartes: textes et débats ", Librairie Générale Française, Paris, 1984, p.391.

THÉMATA. Revista de Filosofía, No 51 enero-junio (2015) pp.: 443-454

doi: 10.12795/themata.2015.i51.25 
qui sont naturellement en nos âmes, et qui sont elles-mêmes déduites ou sensiblement, des principes et de Dieu. [...] Je dirai plus. Nous savons bien qu'il n'eût pas trouvé Dieu [...] s'il n'avait pas eu [...] une certaine expérience de Dieu. [...] Qu'importe. Descartes, dans l'histoire de la pensée, ce sera toujours ce cavalier français qui partit d'un si bon pas ${ }^{9}$ \%. Peguy questionne la solidité des principes de Descartes et lui reproche de partir du haut alors qu'il ne peut avancer concrètement dans sa démarche sans faire référence à l'expérience. Ainsi, même l’idée de Dieu provient d'une «expérience de Dieu».

Au contraire, Merleau-Ponty, veut traiter les questions de l'homme et du monde en partant du bas. Pour ce faire, il introduit la notion de comportement [conduite], commençant sa recherche par les comportements les plus basiques [les réflexes], pour aller vers les comportements les plus subtiles [projection, représentation]. L'auteur souligne l'importance du comportement qui, loin d'être la résultante d'une série de causes purement physiologiques, est avant tout la manière dont l'homme entre en relation avec la vie et le monde environnant. L'expérience chez l'homme révèle tant les mouvements corporels comme les moments du comportement. Le comportement peut être pris alors comme phénomène à partir duquel il peut $\mathrm{y}$ avoir retour. Cela permet de remonter des faits à l'être ${ }^{10}$, «aux postulats ontologiques», suscitant la question suivante chez l'observateur : pourquoi tel comportement? Merleau-Ponty élabore alors une critique on ne peut plus justifiée de la physiologie expérimentale qui est, en réalité, un mécanicisme, mais qui présente également des problèmes de fausseté au niveau de sa méthode, de son propre procédé : au travers de ses théories, elle ne cesse d'anticiper l'interprétation des comportements observés. Ces théories substituent alors la description fidèle du comportement. La phénoménologie pontienne, veut, au travers de la notion de comportement, supprimer l'opposition entre Nature et conscience, entre chose et idée, entre intérieur et extérieur. Il s'agit de revenir au monde vécu, c'est-à-dire de prendre au sérieux le patient et de prendre au sérieux l'expérience originelle. Ainsi, la conscience peut être replacée au niveau de la vie. [Toutefois, cette entreprise, d'après Bimbenet, n'a pas abouti puisque la conscience s'impose comme l'élément dominant de l'homme, comme forme supérieure qui synthétise les autres formes et leur donne leur sens proprement humain ${ }^{11}$. Cela permet à notre auteur d'affirmer que la philosophie de Merleau-Ponty est, dans ces débuts, encore tintée de dualisme $\left.{ }^{12}\right]$.

[9] Ch.Peguy, "Note [...]sur Descartes..., Pléiade, Oeuvre en prose, Gallimard, Paris, 1961, pp.1359-1361.

[10] La démarche de la philosophie mécaniste, mais aussi de la philosophie spiritualiste, ainsi qu'une bonne partie de la psychologie et psychanalyse utilise la méthode déductive appliquant des principes théoriques à la réalité. Au contraire, Merleau-Ponty, au travers de la méthode phénoménologique, veut laisser parler les faits, revenir à la nature.

[11] Bimbenet met en relief le décalage qu'il y a entre le programme de Merleau-Ponty et le résultat final. Merleau-Ponty n'aurait pas réussi à dépasser le dualisme dans ces deux premiers ouvrages.

[12] Nous tenons à nuancer cette affirmation [nuancée de fait dans d'autres articles de l'auteur comme "l'homme ne peut jamais être un animal»]. En effet, d'accord avec la philosophie de la forme,

THÉMATA. Revista de Filosofía, No51 enero-junio (2015) pp.: 443-454

doi: 10.12795/themata.2015.i51.25 
Nature et humanité : évolution de ces concepts dans l'œuvre de Maurice Merleau-Ponty d'après Étienne Bimbenet

Le retour à l'expérience originelle nous découvre l'essence des comportements vivant. La vision scientifique selon laquelle la cause de tout ne serait qu'une série de réactions physico-chimiques (action, stimulation-réaction) n'est que trop lacunaire. Une conduite est vivante lorsqu'elle manifeste une unité indécomposable qui est immédiatement l'indice d'une signification vécue par l'organisme. L'organisme n'est donc pas sensible à des stimulations isolées mais aux propriétés formelles de l'excitant ou aux relations spatio-temporelles. L'organisme réagit à une situation globale nommée par la Gestalt-théorie la forme $^{13}$. Celui-ci comprend la situation en l'imprégnant de significations vitales. On ne parle plus alors d'entourage géographique mais de milieu de comportement. Il s'agit non plus d'une somme d'évènements purement physiques et extérieurs à l'organisme mais d'une situation adaptée par et pour l'organisme en fonction de ses besoins vitaux. Maintenant, du côté des réactions de l'organisme, les gestes de ce dernier pour répondre à une situation particulière évoque également une unité structurale (et non pas une somme de reflexes élémentaires). La conduite possède une cohérence, un ordre interne ; sorte de trajet rempli de signification. Le comportement peut être conçu de deux manières distinctes. D'une part, il y a le comportement géographique, qui est la somme des mouvements effectivement donnés par l'animal en rapport avec le milieu physique. D'autre part, il y a le comportement vital ou vrai, c'est-àdire l'ensemble des mouvements considérés dans leur agencement intérieur (articulation intelligible). Une vision adéquate de l'être vivant, nous amène à considérer l'ensemble des gestes du corps comme impliquant effectivement la conscience de l'observateur. Ceux-ci exigent une compréhension intérieure du sens. La notion de comportement réclame une compréhension de l'intérieur comme un défilé de significations vitales. Le vivant va être compris alors comme une forme intentionnelle de type fonctionnelle.

Dans la nature, nous pouvons distinguer trois types de formes : la matière (domaine physique), la vie (domaine vital) et l'esprit ou conscience (domaine humain). La grande question est la suivante : comment peut-on unir ces trois causalités sans faire référence à une philosophie de la substance ? Pour commencer, il ne faut pas opposer la quantité, l'ordre et la signification présents dans la matière, dans la vie et dans l'esprit. La " scission"

développée par Merleau-Ponty dans la Structure, la conscience est une forme propre de la structure humaine. Cela ne signifie pas que la conscience domine tel le Cogito de Descartes. A l'inverse, le phénoménologue français propose une définition plus large de la conscience qui s'appuie d'abord sur une intentionnalité. La conscience représentative n'est qu'une forme particulière de la conscience qui se définit plutôt comme un réseau d'intentions significatives, vécues plutôt que connues. C'est donc l'action qui sera le véritable reflet de la condition humaine ou de la condition animale.

[13] Distinction structure-forme expliquée de manière éclairante dans le livre de Bimbenet : pages 48 à 61 . Prenons une image selon laquelle la structure serait le tronc constitutif de l'être [la structure peut comporter différents niveaux] et les formes, les modalités d'exercices de cette structure [les niveaux ou branches].

THÉMATA. Revista de Filosofía, No51 enero-junio (2015) pp.: 443-454

doi: 10.12795/themata.2015.i51.25 
dans la nature semble se produire lorsque la conscience apparaît. Un être présente un langage qui n'est plus seulement le signe d'une communication émotionnelle ou pratique mais la marque d'une pensée structuré où se laisse entrevoir un contenu abstrait et catégorisé. Et alors l'attitude catégoriale est le sommet de l'échelle de la nature ${ }^{14}$. Le signe de l'intelligence est la capacité de voir les choses de manière globale, la capacité d'avoir une vue d'ensemble. C'est dans cette potentialité justement que réside la différence structurale entre l'homme et l'animal. Chez l'animal, nous pouvons observer une intentionnalité purement fonctionnelle, liée à un contexte pratique ${ }^{15}$. Par contre chez l'homme, la connaissance est avant tout une perception, c'est-à-dire la capacité de regarder la nature avec profondeur. C'est pourquoi la vision, chez l'homme, est un organe spirituel, capable d'objectiver le spectacle. Le voir humain est un voir perspectif. L'animal ne possède pas cette faculté qui permet de faire des différents moments, au travers de la multiplicité de perspectives, une seule et même chose.

Cette capacité est la marque de la liberté de l'homme, puisqu' il possède la faculté de ne pas être attaché au moment, au présent de la tâche. Il comprend le monde et s'adresse à lui en terme de symboles et significations, au contraire de l'animal qui, lui, a un rapport tout à fait différent avec le monde ; son mode d'entrer en relation avec lui est pratique et se produit grâce à l'intuition. En ce sens, le corps humain se différencie absolument de l'organisme. La main humaine se convertit en un instrument d'instruments (et n'est pas un simple instrument). Le corps de l'homme a une plasticité remarquable, un corps indéfiniment adaptable. De la sorte, Bergson osait affirmer que l'intelligence est la faculté de fabriquer et d'employer des instruments inorganisés. A partir de ces réflexions, nous pouvons conclure deux choses. La première est que l'anthropologie est structurale et aussi que l'anthropologie est inséparable d'une philosophie de la nature, matérielle et vivante. La vie et l'esprit sont deux types de conduites dont une intègre l'autre. Il y a une opposition fonctionnelle entre les deux mais pas substantielle. L'esprit ne vient pas couronner une animalité. Il ne saurait s'agir d'un corps magnifié par une conscience. Nous parlerons de préférence d'une humanisation d'un corps. Cela se reflète dans tous les secteurs de la vie humaine : l'habillement, la sexualité, etc. Dans tous ces domaines une intentionnalité distincte apparaît. Mais alors, l'homme,

[14] Elle est le sommet dans le sens où c'est elle qui permet de thématiser les autres niveaux.

[15] D'autres auteurs contemporains tels que Macintyre ont insisté sur les similitudes entre l'homme et l'animal s'appuyant sur cette intentionnalité pratique ainsi que sur leur capacité de sentir. Selon Macintyre cela serait dû au fait de posséder le même socle commun : le socle biologique [appartenance à la nature]. Merleau-Ponty s'efforce également de partir du bas et de montrer les similitudes entre les deux, mais en insistant intensément sur le fait que le niveau biologique chez l'homme est toujours empreint "d'humanité ". "Tout est fabriqué et tout est naturel chez l'homme ". D'une certaine manière, la faculté de sentir et de vivre la souffrance physique, chez l'homme, se démarque par cette capacité d'investir le légat biologique d'un sens nouveau.

THÉMATA. Revista de Filosofía, Nº51 enero-junio (2015) pp.: 443-454

doi: 10.12795/themata.2015.i51.25 
par son esprit, se sépare-t-il de la nature ? La réponse est négative, car tout est fabriqué et tout est naturel chez l'homme. Celui-ci a une structure unifié. Son comportement est donc emprunt tant d'esprit comme de comportements intégralement biologiques. La vie humaine est ce va et vient de l'existence qui se laisse être tantôt biologique, tantôt personnel. Nous nous référons à la conscience, en conséquence, comme conscience naturée ${ }^{16}$.

\section{Expression et effacement du thème anthropologique}

La conscience naturée est certainement le chapitre central du livre de Bimbenet et correspond au troisième chapitre de la Structure du Comportement. Dans l'ordre humain, elle représente notre appartenance à la nature [par opposition à la conscience naturante ou attitude catégoriale]. Grâce à la notion de comportement, Merleau-Ponty peut poser une communication intérieure entre conscience et action [la structure étant ce qui finit d'enraciner la conscience dans l'agir et d'assurer le lien entre l'homme et la nature]. La méthode phénoménologique permet de retrouver [voir note 15] ce rapport originel avec la nature, ce départ noématique. Bimbenet peut alors donner une première définition de la nature primordiale : "C'est ce que l'homme perçoit, et corrélativement l'acte qui rend compte de ce perçu : le champ phénoménal avant l'intentionnalité ${ }^{17}{ }^{\prime}$. Pour comprendre le sens de la nature en nous, il est utile d'analyser la perception enfantine [dans laquelle la pensée objectivante n'est pas encore vraiment présente $\left.{ }^{18}\right]$. Dans la perception commençante, nous pouvons observer une proximité avec la nature qui prend du sens petit à petit chez l'enfant. De cette façon, l'enfance manifeste le sous-bassement naturel de la raison dans lequel sont visées des intentions humaines plutôt que des objets de la nature ou des qualités pures. En d'autres termes, les objets de la nature, ainsi que les personnes, sont éprouvés par l'enfant plutôt que connus théoriquement. L'enfant perçoit, premièrement, des corps humains [celui de la mère d'abord] et surtout l'expressivité naturelle qui les rend visibles : la physionomie, les gestes, l'intonation de la voix, les expressions du visage. En deuxième instance, il appréhende l'entourage pratique de ces corps, c'est-àdire les objets d'usages qui manifestent une intention pratique : une voiture, une chaise, un lit. ${ }^{19}$ Avant d'être la nature en soi, le monde est vécu par nous de

[16] Alors que les deux premières œuvres semblent refléter une prépondérance de la conscience par rapport au corps, Merleau-Ponty parait toutefois enclin à retrouver ce rapport originel avec la nature ; rapport retrouvé grâce à la méthode phénoménologique.

[17] Etienne Bimbenet, "Nature et humanité: le problème anthropologique dans l'œuvre de Merleau-Ponty ", Vrin, Paris, 2004, p.157.

[18] Chez l'enfant, il y a une unité entre l'empirique et le transcendantal.

[19] Seul l'adulte peut prendre du recul avec le monde environnant et se placer devant lui comme spectateur. Tel est le point de départ de toute science qui se détache du monde et des objets en

THÉMATA. Revista de Filosofía, Nº51 enero-junio (2015) pp.: 443-454

doi: 10.12795/themata.2015.i51.25 
manière humaine, car il est modelé et présenté par et pour l'homme. Le langage, enfin, serait, en troisième lieu, ce qui structure la perception enfantine. Le mot n'est pas d'abord le signal d'un concept [le support] mais il est le moyen qui permet la communication entre deux personnes ; il manifeste un sens pratique. Bimbenet tire donc la conclusion suivante : l'enfant semble aller vers la reconnaissance du caractère originaire de l'expressivité du sensible ${ }^{20}$.

Une notion originale qui vient éclairer la perception enfantine est celle de l'a priori matériel. C'est bien parce que les intentions que l'enfant comprend sont indécomposables qu'elles sont a priori ${ }^{21}$. Mais, étant donné qu'elles appartiennent nécessairement à l'univers perçu, elles sont particularisées par le contexte humain, pratique ou linguistique [elles sont immanentes au déploiement sensible] et donc sont matérielles [d'où la formule a priori matériel]. C'est donc l'expressivité du sensible qui donne le dernier mot à la perception commençante car les significations humaines qui en émergent sont inséparables du contenu sensibles dans lequel elles s'incarnent. La nature primordiale serait donc ce champ sensible préobjectif dans lequel apparaît tout le reste. Dans ce contexte, quel type de conscience peut capter une telle expressivité ? Seule une conscience qui éprouve ou vit ces intentions humaines. Cette conscience est retenue dans une profondeur corporelle ; il s'agit d'une conscience incarnée. Comme nous l'avons mentionné plus haut, la conscience se définit avant tout par son intentionnalité ; intention qui vise des objets de plusieurs types : affectifs, sensibles, intellectuels, etc. ${ }^{22}$ La conscience peut donc être affective, volitive, représentative, désirante. Surgit alors une question que Bimbenet exprime de la manière suivante : quel est le point commun entre les différentes consciences ? Le point de départ de toute expérience est ce socle commun, soit le champ sensible préobjectif auquel la conscience se rapporte. Reprenant les propos de Max Scheler, notre auteur affirme ainsi que la conscience à laquelle fait référence Ponty est en premier lieu pratique [car elle est une conscience de milieu]. Il n'y a d'autre manière de percevoir un milieu que de se sentir concerné par lui, ou de communiquer intérieurement avec lui sous la forme d'une épreuve. Nous sommes face à une intériorité vitale, une communication

général pour en offrir une explication théorique.

[20] Bimbenet fait référence, dans une de ses notes de bas de page [page 162] à une remarque de Paul Guillaume qui insiste sur le fait que " dès le début, l'homme est pour l'enfant l'objet intéressant par excellence" (L’imitation chez l'enfant. Etudes psychologiques, F.Alcan, Paris, 1925, p.122). L'homme devient ainsi pour l'enfant " la mesure de toutes choses ". Nul doute que cet auteur ait influencé la pensée de Merleau-Ponty pour qui la psychologie enfantine révèle bien des choses.

[21] A priori: qui ne peut pas être conçu partie par partie et qui doit être pensé comme essence indécomposable.

[22] La conscience se présente comme un réseau d'intentions significatives. Il y a plusieurs manières pour la conscience de viser son objet et plusieurs sortes d'intentions en elle.

THÉMATA. Revista de Filosofía, No51 enero-junio (2015) pp.: 443-454

doi: 10.12795/themata.2015.i51.25 
intérieure entre un sujet et un milieu particulier, reflet de l'unité originaire du sentant et du sensible ; sentant qui s'exprime par un langage et une corporéité et sensible qui s'exprime par sa visibilité, sa matérialité. Tel est le sens de la conscience naturée. La nature primordiale est, finalement, cette intériorité même, cette intimité de l'organisme percevant et de son monde ; ou plutôt d'intériorisation gagnée sur l'extériorité même.

A partir de la Prose du monde [début des années 50], il semble que Merleau-Ponty donne une nouvelle tournure à sa philosophie en «déplaçant le regard vers le haut». En effet, il s'intéressera alors au champ de la connaissance à proprement dit : le monde de l'expression [par opposition au monde sensible] ${ }^{23}$. Selon Bimbenet, la Prose $d u$ monde touche donc directement au statut de notre humanité en tant qu'humanité. Une nouvelle philosophie est entamée qui prend comme point d'appui les pouvoirs d'expression du langage. La fonction du langage peut être comprise d'après l'image du peintre et de son œuvre. Le peintre exprime une réalité éprouvée, observée ou imaginée. Ainsi, Monnet nous offre de merveilleux paysages mais depuis une perspective impressionniste qui nous dévoile également la puissance créatrice de son auteur. Le tableau devient une interprétation, une re-création. Nous utilisons la parole re-création car le pouvoir créateur lui vient d'une puissance d'expression plus ancienne et originaire : celle du visible. "C'est de la matière silencieuse du monde que le peintre tire sa gloire ${ }^{24}{ }^{4}$. Le phénoménologue français pense que cette expressivité repose sur le dynamise du sensible. Le sensible doit donc se comprendre selon une modalité dynamique. Ce que nous percevons, c'est le mouvement de la chose ; c'est lui encore qui nous touche, nous impacte et nous invite à l'exprimer. Les différentes réductions phénoménologiques et l'intérêt croissant de Ponty envers le thème de la Nature, conduit son œuvre, vers un effacement progressif du thème anthropologique.

En conclusion, les outils proposés par Merleau-Ponty tout au long de ses recherches sont l'observation et la description. En ce sens, nous pouvons observer que l'homme se démarque sans cesse de la nature par son rapport particulier au monde ; rapport défini par l'apparition de la conscience. Seul l'homme est capable de voir le général dans le particulier ; l'essence dans le sensible. Mais cette dialectique spirituelle s'incarne nécessairement dans une dialectique vitale. "On n'agit pas avec l'esprit seul». En somme, tout est art et tout est naturel en l'homme. Il n'y a pas une conduite qui ne trouve quelque source dans l'être biologique tout en s'y détachant pour se convertir en un acte proprement humain. Tel est le mouvement dialectique de l'existence : un va-etvient insensible de la vie naturelle à la vie de l'esprit. Le corps est ce véhicule

[23] Par expression, Bimbenet comprend avant tout le langage [langage corporel, paroles, etc.]. Merleau-Ponty s'inspire fondamentalement des travaux de De Saussure.

[24] « Nature et humanité», p.217.

THÉMATA. Revista de Filosofía, Nº51 enero-junio (2015) pp.: 443-454

doi: 10.12795/themata.2015.i51.25 
qui à la fois nous ouvre au monde, nous rappelle notre lien de parenté avec la nature et nous permet d'exprimer concrètement les actes les plus humains, les idées les plus géniales, l'art dans toutes ses facettes. Il ne s'agit pas d'un esprit ajouté à un corps, mais d'une autre manière d'être corps pour l'homme. L'esprit ne sublime pas le corps afin de le faire obéir à ses desseins, mais c'est le corps qui permet l'avènement de l'esprit. Cette autre manière d'être corps est possible, souligne Bimbenet, car le corps humain est plus que corps dans le sens d'organisme, il est chair. Il semble, toutefois, que Merleau-Ponty veuille éviter une métaphysique qui s'appuierait sur des concepts universels et intemporels comme nature, finalité ou raison car ces notions se discernent comme hors du temps et de l'histoire. Une philosophie de la vie ou de l'être considère l'humain comme un sujet qui s'incarne dans le mouvement de l'histoire. Étienne Bimbenet nous entraîne, donc, au fil de l'œuvre de Merleau-Ponty, de concepts en concepts afin de saisir la relation existante entre Nature et Humanité. Ainsi, son livre se convertit en un résumé conséquent et intéressant du Corpus Pontien, bien que parfois difficile à lire à cause d'un langage très ou peut-être trop expérimenté. 